\title{
Risks of infection, hospital and ICU admission, and death from COVID-19 in people with asthma: systematic review and meta-analyses
}

Afolarin Otunla, BA, Medical Sciences Division, University of Oxford, Oxford, Oxfordshire, UK

Karen Rees, PhD. Freelance systematic reviewer, Warwickshire, UK.

Paddy Dennison, PhD, MRCP, University Hospitals Southampton NHS Foundation Trust, Southampton, UK.

Richard Hobbs, FMedSci, Nuffield Department of Primary Care Health Sciences, University of Oxford, UK.

Jana Suklan, PhD, NIHR Newcastle In Vitro Diagnostics Co-operative, Newcastle University, Newcastle, United Kingdom

Ella Schofield BM BCh, Medical Sciences Division, University of Oxford, UK.

James Gunnell BM BCh, Medical Sciences Division, University of Oxford, UK.

Alexandra Mighiu, DPhil, Medical Sciences Division, University of Oxford, UK.

Jamie Hartmann-Boyce (corresponding author), DPhil, Nuffield Department of Primary Care Health Sciences, University of Oxford, UK. Jamie.hartmann-boyce@ phc.ox.ac.uk; 01865289 206. Radcliffe Primary Care Building, Radcliffe Observatory Quarter, Woodstock Road, Oxford, UK, OX2 6GG. 


\section{$\underline{\text { Abstract }}$}

\section{Objectives}

To determine if and to what degree asthma may predispose to worse COVID-19 outcomes in order to inform treatment and prevention decisions, including shielding and vaccine prioritisation.

\section{Design}

Systematic review and meta-analysis.

\section{Setting}

Electronic databases were searched (October 2020) for clinical studies reporting at least one of the following stratified by asthma status: risk of infection with SARS-CoV-2; hospitalisation, intensive care unit (ICU) admission, or mortality with COVID-19.

\section{Participants}

Adults and children who tested positive for or were suspected to have COVID-19.

\section{Main outcome measures}

Main outcome measures were the following stratified by asthma status: risk of infection with SARS-CoV-2; hospitalisation, intensive care unit (ICU) admission, or mortality with COVID-19. We pooled odds ratios (ORs) and presented these with $95 \%$ confidence intervals (CIs). Certainty was assessed using GRADE.

\section{Results}

$30(n=112,420)$ studies were included (12 judged high quality, 15 medium, 3 low). Few provided indication of asthma severity. Point estimates indicated reduced risks in people with asthma for all outcomes, but in all cases the evidence was judged to be of very low certainty and $95 \%$ CIs all included no difference and the possibility of increased risk (death: OR 0.90, 95\%CI 0.72-1.13, $\mathrm{I}^{2}=58 \%$; hospitalisation: OR $0 \cdot 95,95 \%$ CI $0 \cdot 71-1 \cdot 26$; ICU admission: OR $0 \cdot 96,95 \%$ CI $0 \cdot 75-1 \cdot 24)$. Findings on hospitalisation are limited by substantial unexplained statistical heterogeneity. Within people with asthma, allergic asthma was associated with less COVID-19 risk and concurrent COPD was associated with increased risk. In some studies, corticosteroids were associated with increased risk, but this may reflect increased risk in people with more severe asthma.

\section{Conclusions}

Though absence of evidence of a clear association between asthma and worse outcomes from COVID-19 should not be interpreted as evidence of absence, the data reviewed indicates that risks from COVID-19 in people with asthma, as a whole, may be less than originally anticipated.

Registration: https://osf.io/c9e6d/ 
Trial registration

Not applicable.

$\underline{\text { Summary }}$

\section{What is already known about this subject?}

- At the beginning of the pandemic, it was assumed that people with asthma (PWA) would be at increased risk of poor outcomes with COVID-19.

- This was contradicted by early primary studies, which reported that PWA represented a lower proportion of those admitted to hospital with COVID-19 than seen in the general population.

- Systematic reviews and meta-analyses on this topic have generated conflicting conclusions, with some finding an association between asthma and poor COVID-19 outcomes whilst others do not. Analysis of the quality of these reviews reveals significant pitfalls, ranging from incomplete risk of bias reporting to inadequate justification for exclusion of individual studies.

\section{What are the new findings?}

- This systematic review is one of the largest to date on the effects of asthma on the risk of poor COVID-19 outcomes, covering a large number of studies across multiple continents.

- Pooled results showed that, overall, asthma was not associated with severe COVID19 outcomes, but evidence was judged to be of very low certainty.

How might it impact clinical practice in the forseeable future?

- Evidence suggests that PWA are not at increased risk of acquiring SARS-CoV-2 compared to those without asthma, and have similar, if not slightly improved, clinical outcomes with COVID-19.

- However, absence of evidence should not be interpreted as evidence of absence, and further high quality primary studies are required to reinforce this conclusion. 


\section{Introduction}

The severe acute respiratory syndrome coronavirus 2 (SARS-Cov-2) pandemic has affected over 158 million people worldwide, with at least 3.28 million deaths due to COVID-19 as of $9^{\text {th }}$ May 2021. ${ }^{1}$ At the start of the pandemic, people with asthma (PWA) were assumed to be at increased risk from COVID-19, as respiratory viral infections are well known to cause severe adverse outcomes in patients with established asthma through stimulation of exacerbation episodes. ${ }^{2}$

Early, large-scale case series provided evidence to the contrary, reporting that PWA made up a lower proportion of patients admitted to hospital with COVID-19 than seen in the general population. ${ }^{3-5}$ These findings birthed a myriad of hypothesised immunopathological mechanisms to explain this apparent reduction in susceptibility to severe COVID-19. ${ }^{6,7}$ More recent epidemiological studies have further complicated our understanding of the association between COVID-19 and asthma, with some suggesting an association whilst others do not. ${ }^{8-}$ ${ }^{15}$ It is important for PWA, their carers, healthcare providers, and policymakers to understand if and to what degree asthma may predispose to worse COVID-19 outcomes. Such information impacts both treatment and prevention decisions, including shielding and vaccine prioritisation.

The ambiguity of primary evidence combined with the clear importance of determining the relationship between COVID-19 and asthma has resulted in a number of systematic reviews on the subject ${ }^{16-18}$. Analysis of the quality of these reviews reveals significant pitfalls, ranging from incomplete risk of bias reporting to inadequate justification for exclusion of individual studies. This is reflected in the scientific brief on Asthma and COVID-19 released by the World Health Organisation (WHO), in which only 1 of 6 systematic reviews on the subject were judged to be free of 'critical weakness' using the AMSTAR-2 ratings for critical domains. ${ }^{19}$ The single high quality review identified through the WHO scientific brief focused on mortality and concluded that there was insufficient evidence to draw firm conclusions. ${ }^{18}$ The review contained published literature up to June $8^{\text {th }} 2020$, and further studies have since emerged. In addition, no systematic reviews of sufficient quality have examined whether people with asthma are more likely to be infected with COVID-19 or be hospitalised or admitted to intensive care with COVID-19 than people without asthma.

We therefore conducted a comprehensive systematic review and meta-analysis of published literature to determine whether asthma is a risk factor for worse outcomes in both adults and children with COVID-19, focusing on the specific endpoints of infection, hospitalisation, intensive care unit (ICU) admission and mortality. We also investigated whether, within people with asthma, asthma medication, severity, comorbidity, age and ethnicity affected COVID-19 outcomes.

\section{Methods}

\section{Search Strategy}

This systematic review and meta-analysis followed the Preferred Reporting Items for Systematic Reviews and Meta-Analysis (PRSIMA) guidelines, and incorporates the PRISMA-Equity extension designed for systematic reviews with a focus on health equity. ${ }^{20}$ We were commissioned by the World Health Organization (WHO) to conduct a rapid review on this topic, the protocol for which was specified and made publicly available before review 
conduct. ${ }^{21}$ This review follows on from that work; our WHO review did not include statistical synthesis, and therefore following the WHO rapid review, we posted a new protocol outlining our synthesis plans, results from which are presented here (https://osf.io/c9e6d/).

We searched Medline, Embase, LitCOVID and the Cochrane study register on 8 October 2020 for COVID-19 clinical studies, published or accepted for publication but not yet published, in any language. We included any type of clinical study (randomised controlled trials, cohort studies, cross sectional studies, case reports and series and case-control studies) that reported the number of adults or children with asthma (as determined by the investigators) and COVID-19 (either confirmed with RT-PCR test or suspected), and specified at least one of the following outcomes stratified by asthma status: risk of infection; hospitalisation status; ICU admission status; mortality. Studies were excluded if they did not report any of our pre-specified outcomes in people with asthma and COVID-19. The full search strategy is outlined in appendix 1 (pp 2-4). Two reviewers independently screened titles and abstracts, and full texts of selected references, with discrepancies resolved by discussion or referral to a third reviewer. Where grey literature was identified, we contacted the authors where it was unclear if grey literature had been accepted for publication; if it had been (i.e. had been subject to peer review) then we included it in our review. Non-peer reviewed publications were excluded, as specified in advance.

Two reviewers independently assessed methodological quality using the Newcastle-Ottawa Scale for cohort and cross-sectional studies. The Newcastle-Ottawa scale consists of 3 domains across which 9 points are available (9 for cohort studies, 6 for cross-sectional studies); selection of study groups (4 points), comparability of groups ( 2 points) and ascertainment of exposure and outcomes (3 points). ${ }^{22}$ We considered a score of 1-3 stars as low quality, 4-6 as medium quality and 7-9 as high quality. We also rated the certainty of the evidence according to the GRADE approach ${ }^{23}$, which we present in a summary of findings table (Table 2). Two reviewers independently extracted data on patient characteristics and clinical outcomes using a bespoke extraction form which was piloted before use to facilitate efficient extraction of data. Any discrepancies were resolved by discussion. When a study presented asthma patients as a percentage of total population, we estimated the number based on the given percentage.

\section{Data analysis}

We performed meta-analyses using the Cochrane Review Manager 5.4 platform to determine the risk of infection with SARS-CoV-2, ${ }^{24}$ hospitalisation with COVID-19, ICU-admission with COVID-19 and risk of mortality from COVID-19 in people with asthma vs those without asthma, presenting results as odds ratios (ORs) with $95 \%$ confidence intervals (CIs). We used prevalence of asthma in COVID-19 patients as a proxy for risk of infection, although we appreciate this is significantly limited by lack of widespread random testing. In each meta-analysis, we fitted a random-effects model using the inverse-variance method, using ORs as reported within the study or calculated based on data provided in accordance with the Cochrane Handbook. ${ }^{25}$ When producing the prevalence meta-analysis, we used prevalence ratios and calculated the $95 \%$ CIs in accordance with the Cochrane Handbook. When studies reported both adjusted and unadjusted estimates, we used the adjusted estimates and performed subgroup analysis to compare adjusted and unadjusted values. We assessed heterogeneity using the Cochrane $\mathrm{I}^{2}$ value, and used their suggested thresholds for moderate, substantial and considerable heterogeneity. ${ }^{26}$ We undertook sensitivity analysis removing studies at high risk of bias. The following pre-specified subgroup analyses were 
performed: outcomes by mean/median age (up to 60 years and 60 years and above), by mean/median BMI (less than and greater than 30), by ethnicity (less than and greater than $50 \%$ white), by asthma severity (as defined by each study), by asthma medication and by adjustment status (studies where the effect size was adjusted for factors including age, sex, BMI and comorbidities compared to studies which did not adjust at all). We visually inspected the funnel plots for all comparisons with over 10 studies for possible publication bias. In meta-analyses with less than 10 studies, we performed Egger analysis.

We narratively report results from studies that could not be included in meta-analysis. We were also interested to know whether asthma medication, severity, comorbidity, age and ethnicity affected COVID-19 outcomes in people with asthma. We narratively report results from studies assessing the extent to which different characteristics within people with asthma predispose to our outcomes of interest, using effect direction plots. ${ }^{27}$

\section{$\underline{\text { Results }}$}

A total of 824 articles were identified from the search of databases. The selection process is presented in a PRISMA flow diagram (Figure 1). After initial screening based on titles and abstracts, 97 articles remained for full text evaluation. Full text analysis produced a total of 30 studies which met the inclusion criteria, 25 of which had sufficient data to be included in meta-analysis.

Of the 30 studies included $\left({ }^{9-12,17,18,28-51}\right)$ totalling 112,420 people who tested positive for or were suspected to have COVID-19, there were 24 cohort studies (22 conducted retrospectively and 2 conducted prospectively) and 6 cross-sectional analyses. All were published after peer review and no pre-prints were included in the analyses. Sample sizes ranged from 106 to 818,490 people, with median sample size $n=1043$ and IQR=7145 (445 7590). Studies were from North and South America (12), Europe (14) and Asia (4). The majority of studies used adult (18+) cohorts (19), 6 studies used mixed paediatric and adult cohorts and 1 study used a paediatric cohort. The remaining studies did not report sufficiently detailed age data to determine whether children were included. Only one study included people with suspected but unconfirmed SARS-COV-2; this study reported only on prevalence and was not included in meta-analyses ${ }^{47}$. We assessed studies for possible overlap to ensure participants were not being double-counted in our analyses; studies were conducted in different cohorts (e.g. different hospitals) with no overlap identified.

Using the Newcastle-Ottawa scale, 12 studies were rated as high quality, 15 studies as medium quality and 3 studies as low quality (overall NOS scores for each study can be seen in supplementary Table 1, scores for individual domains can be seen in appendix 1, Supplementary Table 2). A summary of included studies is presented in supplementary Table 1.

\section{Outcomes}

\section{Prevalence}

21 studies reported prevalence of asthma in those suffering from COVID-19. The estimated prevalence of asthma in patients with COVID-19 ranged from $0 \cdot 32 \%$ to $25 \cdot 8 \%$ (Median 
9.14\% and IQR 11.15 (3.05-14.2)). We included all of these studies in meta-analysis. Data was highly heterogeneous. The pooled prevalence of the 21 studies was $9.38 \%$ (95\% confidence interval 7·38-11·38, $\left.\mathrm{I}^{2}=99 \%, \mathrm{n}=83,294\right)$. Among these studies, there was some asymmetry to the funnel plot, raising the possibility of publication bias (Supplementary Figure 1). Overall, this evidence was judged to be of very low certainty due to unexplained statistical heterogeneity and possible publication bias.

In order to provide a more contextualised view, we compared asthma prevalence in each study population to prevalence in the general population of the country in which the study was based (Figure 2).

We looked to clinical study setting (whether hospital based, or community based) as a potential driving force behind the observed heterogeneity. However, high heterogeneity was maintained in subgroup analysis stratifying studies based on whether their cohorts were hospital based, community based or mixed $\left(\mathrm{I}^{2}=99 \%\right.$ in both hospital and mixed subgroups; test for subgroup differences $I^{2}=55 \%$, Supplementary figure 2 ).

Further subgroup analysis provided evidence that BMI (test for subgroup differences $\mathrm{I}^{2}=$ $60.4 \%$, supplementary figure 3 ) and ethnicity (test for subgroup differences $\mathrm{I}^{2}=94.9 \%$, supplementary figure 4) may have contributed to some of the variation. This was a contrast to subgroup analysis by age (supplementary figure 5), where no subgroup differences were apparent $\left(I^{2}=0 \%\right)$.

We performed sensitivity analyses to assess risk of bias by removing high risk of bias studies (0-3 on the Newcastle-Ottawa scale). Niquini et al was the only high risk of bias study reporting prevalence outcomes, with removal leading to a slight increase in pooled prevalence from $9 \cdot 38\left(\right.$ CI $\left.7 \cdot 38-11 \cdot 38, \mathrm{I}^{2}=99 \%, \mathrm{n}=83294\right)$ to $9 \cdot 81$ (CI 7.92-11.71, $\mathrm{I}^{2}=99 \%$, $\mathrm{n}=51332$ ), and no impact on heterogeneity. ${ }^{45}$

\section{Mortality}

Mortality data was reported by 15 studies. A meta-analysis of data from 13 of these studies was conducted to evaluate the association between asthma and risk of mortality in patients with COVID-19 (Figure 3). The pooled effect estimate demonstrated that asthma was associated with slightly reduced odds of mortality (OR $0 \cdot 90,95 \%$ confidence interval (CI) $0 \cdot 72-1 \cdot 13, \mathrm{I}^{2}=58 \%, \mathrm{n}=62521$ ), although CIs were wide and incorporated no difference as well as increased risk. The funnel plot analysis of these studies did not suggest publication bias (supplementary figure 6). There were no high risk of bias studies within this outcome, therefore we did not perform a sensitivity analysis. We judged this finding to be of very low certainty due to unexplained statistical heterogeneity and imprecision (wide CIs).

Subgroup analyses by age (supplementary figure 7) and comparing adjusted versus unadjusted estimates (supplementary figure 8) did not explain the moderate heterogeneity observed. However, there was some evidence that both BMI (test for subgroup differences $\mathrm{I}^{2}$ $=85 \%$ ) and ethnicity (test for subgroup differences $\mathrm{I}^{2}=77 \%$ ) may explain some of the variation. In the 3 studies where the majority of the cohort had a BMI of 30 or over, asthma was associated with an increased odds of mortality, with CIs excluding no difference (OR $1 \cdot 24$, CI $1-1 \cdot 53, n=2951$ ) (Figure 3), but the majority of studies did not provide BMI data. In the 5 studies where the majority of the cohort was of non-white ethnicity, asthma was associated with a reduced odds of mortality, with confidence intervals excluding no difference (OR 0.75, CI 0.60-0.93, n=41,894) (supplementary figure 9). Again, the majority of studies did not report data on ethnicity.

The two studies not included in the meta-analysis (Abrams, 2020 and Beurnier, 2020) were excluded due to the fact that neither reported mortality rates in people without asthma, and 
thus no odds ratio could be extracted or calculated. ${ }^{28,32}$ However, through utilisation of mortality rates reported in hospitalised patients without asthma in other large scale studies over a similar time period, the data can still be interpreted narratively. Abrams reported a mortality rate (percentage of people with asthma who died from COVID-19) of $4.5 \%$ in New York City (NYC) hospitals, which is lower than the $32.1 \%$ mortality rate (percentage of people who died from COVID-19) in NYC hospitals reported by Thompson et al. ${ }^{52}$ Beurnier reported an in-hospital mortality rate (percentage of people with asthma who died from COVID-19) of $8.1 \%$ in Paris, France, which was lower than the in hospital mortality rate (percentage of people who died from COVID-19) of $16.9 \%$ recorded by Gaudart across a similar time period. ${ }^{53}$

\section{Hospitalisation}

9 studies reported hospitalisation in PWA with COVID-19. Meta-analysis revealed that asthma was associated with a slight reduction in odds of hospitalisation with COVID-19 (OR $0 \cdot 95$, CI $0 \cdot 71-1 \cdot 26, n=25,065, I^{2}=94 \%$ ) (Figure 4), although CIs incorporated no difference as well as increased risk. Statistical heterogeneity was high, with some studies showing statistically significantly reduced risk and others showing statistically significant increases in risk. Egger analysis of these studies did not suggest publication bias (Test for funnel plot asymmetry, $\mathrm{p}=0.384$ ). There were no high risk of bias studies within this outcome, therefore we did not perform a sensitivity analysis. We judged this finding to be of very low certainty due to unexplained statistical heterogeneity and imprecision (wide CIs).

There was no evidence that ethnicity, BMI, or whether studies presented adjusted or unadjusted estimates explained the heterogeneity observed $\left(\mathrm{I}^{2}=0 \%\right.$ for all tests for subgroup differences, supplementary figures 10-12). There was also little evidence that sample age affected heterogeneity $\left(\mathrm{I}^{2}=10 \cdot 8 \%\right.$, Figure 4$)$.

\section{ICU admission}

Data on ICU admissions were reported by 9 studies. Meta-analysis of 8 of these studies demonstrated that asthma was associated with a slight reduction in odds of ICU admission with COVID-19 (OR 0.96, CI 0.75-1·24, $\mathrm{n}=13,092, \mathrm{I}^{2}=0 \%$ ) (Figure 5), but CIs included no difference and increased risk. There was no evidence of statistical heterogeneity. Egger analysis of these studies did not suggest publication bias (Test for funnel plot asymmetry, $\mathrm{p}=0.6142$ ). There were no high risk of bias studies within this outcome, therefore we did not perform a sensitivity analysis. We also judged this finding to be of very low certainty due to unexplained statistical heterogeneity and imprecision (wide CIs).

There was no evidence of subgroup differences based on age, BMI, or whether studies presented adjusted or unadjusted estimates $\left(\mathrm{I}^{2}=0 \%\right.$ for subgroup differences in all cases, supplementary figures 13-15). There was little evidence of subgroup difference by ethnicity $\left(\mathrm{I}^{2}=7 \cdot 5 \%\right.$, Figure 5).

The study not included in the meta-analysis (Chibba, 2020) did not report ICU admission in people without asthma, and thus no odds ratio could be extracted. However, through utilisation of national ICU-admission data the data can still be interpreted narratively. Chibba reported that $8.6 \%$ of PWA hospitalised with COVID-19 were admitted to ICU in the USA. 
This is lower than the national average rate of ICU admission in people hospitalised with COVID- $19 ; 32 \% .^{54}$

\section{Do COVID-19 outcomes in PWA differ based on population characteristics?}

Very few studies were sufficiently powered to detect a difference by subgroups within PWA. Data regarding our pre-specified characteristics reported in more than one study can be found in Table 1.

Data on inhaled corticosteroid (ICS) use were not consistent across studies, but the largest study of its type found high dose ICS was associated with higher risk of COVID-19 mortality when compared to SABA.$^{49} \mathrm{~A}$ second study using the same data set found OCS use was also associated with greater risk in people with asthma; ${ }^{8}$ no other studies evaluated OCS use. With the exception of the one study comparing ICS to SABA, ${ }^{49}$ no significant associations were found between SABA and LABA and disease outcomes. Three studies reported on allergic asthma, reporting reduced prevalence ${ }^{9,55}$ and hospitalisation ${ }^{37}$ compared to non-allergic counterparts. Two studies investigated the importance of co-morbid COPD, finding it was associated with increased hospitalisation, ICU admission, mortality ${ }^{18}$ and prevalence ${ }^{37}$. Only one study reported on ethnicity ${ }^{18}$, with univariate analyses using white ethnicity as a reference group finding that all other ethnic groups had higher risks of hospitalisation. The association was statistically significant for Black and Asian groups and remained so in an age-stratified multivariate logistic regression. Only one study looked at BMI. ${ }^{38} \mathrm{Kim} 2020$ found that the association between asthma and mortality increased in those with BMI $\leq 25$; it was unclear if this association was statistically significant.

Regarding the influence of asthma medication on COVID-19 outcomes in PWA, where associations were detected, it was unclear if these were due to mechanisms of the medications themselves, or due to the association between prescriptions for these medications and asthma severity. ${ }^{49}$ People with allergic asthma seem at lower risk of severe outcomes from COVID19 than those with non-allergic asthma, ${ }^{37}$ but again more data are needed to confirm this. People with COPD and asthma appear at higher risk from severe COVID-19 outcomes than PWA who do not have COPD ${ }^{18 \cdot}$ In PWA, risk from COVID-19 appears to increase with age, ${ }^{18,38,43}$ as in the general population.

\section{$\underline{\text { Discussion }}$}

Our systematic review contains 30 studies and to the best of our knowledge is one of the largest to date on the effects of asthma on the risk of poor COVID-19 outcomes, covering a large number of studies across multiple continents. Whether PWA are at increased risk of infection or severe outcomes from COVID-19 remains unclear, with point estimates reporting a slightly reduced risk for hospitalisation, ICU admission, and death with COVID-19 in people with asthma compared to people without asthma, but $95 \%$ CIs encompassing no difference and as well as moderately increased and moderately decreased risk. Our results revealed a $9.38 \%$ prevalence of asthma in those who tested positive for COVID-19, similar to the prevalence of self-reported asthma symptoms globally of $8.6 \%$. Findings were judged to be very low certainty across all outcomes using GRADE criteria, with key limitations being imprecision (with CIs incorporating both benefit and harm in all instances), possible publication bias (in one instance), and unexplained statistical heterogeneity (Table 2.) The 
effect of ethnicity or BMI on the relationship between asthma and COVID-19 outcomes was not well-reported, with only one study reporting on each variable, both of which were low powered. Further investigation into the influence these factors have on COVID outcomes is required, especially when the association between $\mathrm{BMI}^{56,57}$, ethnicity ${ }^{58}$ and poor COVID outcomes in other chronic diseases such as diabetes is considered.

The use of ICS in COVID-19 has been subject to intense scrutiny, due to their potential to both reduce anti-viral immunity juxtaposed with evidence suggesting they may reduce replication of SARS-CoV-2. ${ }^{59,60}$ Though some high quality studies in our review find that corticosteroids are associated with worse outcomes, these are likely confounded, with Schultz stating that the harmful association could be explained by health differences not recorded in the OpenSAFELY database from which information was obtained, most notably, asthma severity. ${ }^{49}$ They reported an incomplete assessment of exacerbation history, a confounding variable which post-hoc analysis demonstrated had one of the greatest impacts on effect size. This, combined with the fact that ICS use will be higher in people with more severe disease, suggests that ICS use does not influence the likelihood of severe COVID-19.

The PRINCIPLE trial has recently shown that inhaled budesonide, a common corticosteroid used in asthma management, shortens recovery times in COVID-19 patients over the age of $50 \cdot{ }^{61}$ Current guidelines suggest ICS use in PWA should be continued until more evidence is available. ${ }^{62}$

There were limitations to this systematic review. Some analyses were limited by significant heterogeneity, even when sub-grouped by geographical region, age, BMI and study quality. This may be a reflection of the fact that sub-group analyses are a relatively crude tool with which to investigate the cause of heterogeneity, as they rely on study level aggregates as opposed to individual characteristics, and are thus prone to confounding factors. It would be more informative to look at individual data in a large, well reported study, the likes of which are not yet available in the literature. The diagnosis of asthma in primary studies was based largely on self-report or physician diagnosis, not using more objective measures such as spirometry or peak flow. This, combined with the tendency for asthma to flip between under and over diagnosis depending on where you are in the world, ${ }^{63}$ has the potential to confound results. Furthermore, factors which influence COVID-19 outcomes, such as obesity, may contribute to asthma misdiagnosis and thus further confound the issue, particularly in those studies not reporting on BMI ${ }^{64}$. However, the same possible causes of confounding exist in other areas, and yet the lack of consistency observed for the relationship between asthma and COVID-19 is in contrast to other long term conditions, notably diabetes where available research suggests clear associations of poorer outcomes. ${ }^{65}$ When we compared unadjusted and adjusted estimates, there was no evidence of difference between the two. If data and reporting in this area improve, future reviews may wish to specify minimal sets of adjustment factors for inclusion.

Incomplete data and reporting bias are also potential issues. We cannot rule out publication bias and the small number of studies contributing to each analysis mean methods for testing for publication bias are underpowered in our sample. As prespecified with WHO, we did not include preprints, which may mean some relevant evidence has been overlooked. Most of the studies reported in our review derive their data from hospitals, where multiple biases are possible; e.g. studies which find higher rates of COVID-19 hospitalisation in PWA could be because PWA are more likely to contract COVID-19, or because if having contracted COVID-19 PWA are more likely to require hospitalisation or to be referred to hospital as a precautionary measure; studies which find lower rates of asthma in people hospitalised with 
COVID-19 could be because PWA have practiced increased protective measures, e.g.

shielding, or because of incomplete recording of asthma status. In primary studies which seek to evaluate risk factors for COVID-19, authors may evaluate long lists of possible predictors, but in their results only highlight those where statistically significant differences are found. We used NOS, the most commonly used critical appraisal tool for observational studies, though its validity has been questioned ${ }^{66,67}$. However, there is no agreed tool for use in this sort of study, and we pre-specified use of NOS in agreement with the WHO. There may also be more recent studies published beyond our search date which we have missed. None of the included studies evaluated the risk of persistence of COVID-19 symptoms (Long COVID) in PWA. ${ }^{68}$ Finally, of the included studies exclusively in PWA, only one high quality study looked at possible associations between asthma severity and death with COVID-19; it found none. Given the paucity of data, we cannot conclude that asthma is not a risk factor for poor COVID-19 outcomes independent of asthma severity.

In conclusion, pooled results do not suggest that PWA are at increased risk for acquiring SARS-CoV-2 compared to those without asthma, and suggest that PWA have similar clinical outcomes with COVID-19. However, results were limited by imprecision, a lack of reporting on asthma severity, and in some cases substantial unexplained statistical heterogeneity. Though the absence of evidence of a clear association between asthma and worse outcomes from COVID-19 should not be interpreted as evidence of absence, particularly given the paucity of data on asthma severity, the data reviewed does give some indication that risks in PWA, as a whole, may be less than originally anticipated. Further high-quality primary studies are required to reinforce and broaden this conclusion in order to facilitate the development of evidence-based shielding and avoidance measures, as well as appropriate vaccination schedules. 


\section{Contributorship statement}

JH-B conceived the original review. AO, JH-B, JS, ES, JG, AM, and KR screened the studies and extracted data. AO drafted the manuscript and performed statistical analysis with guidance from JH-B. RH, PD, KR and JH-B provided expert opinion. All authors contributed to and approved the final manuscript. All authors had full access to all the data in the study. $\mathrm{JH}-\mathrm{B}$ is responsible for overall content as guarantor.

\section{Declaration of interest}

We declare no competition of interests.

\section{Funding information}

Funding from the World Health Organization supported screening, data extraction and quality assessment as part of a original rapid review. There was no direct funding source for this full systematic review and meta-analysis, therefore there is no grant/award number.

\section{Data Sharing}

Data sharing not applicable as no datasets were generated and/or analysed for this study. All data are from publicly available documents, and references are provided should readers wish to look at original sources. 


\section{References}

1. Dong, E., Du, H., \& Gardner, L. (2020). An interactive web-based dashboard to track COVID-19 in real time. In The Lancet Infectious Diseases (Vol. 20, Issue 5, pp. 533 534). Lancet Publishing Group. https://doi.org/10.1016/S1473-3099(20)30120-1

2. Busse, W. W., Lemanske, R. F., \& Gern, J. E. (2010). Role of viral respiratory infections in asthma and asthma exacerbations. In The Lancet (Vol. 376, Issue 9743, pp. 826-834). Elsevier B.V. https://doi.org/10.1016/S0140-6736(10)61380-3

3. Grasselli, G., Zangrillo, A., Zanella, A., Antonelli, M., Cabrini, L., Castelli, A., Cereda, D., Coluccello, A., Foti, G., Fumagalli, R., Iotti, G., Latronico, N., Lorini, L., Merler, S., Natalini, G., Piatti, A., Ranieri, M. V., Scandroglio, A. M., Storti, E., ... Pesenti, A. (2020). Baseline Characteristics and Outcomes of 1591 Patients Infected with SARSCoV-2 Admitted to ICUs of the Lombardy Region, Italy. JAMA - Journal of the American Medical Association, 323(16), 1574-1581. https://doi.org/10.1001/jama.2020.5394

4. Richardson, S., Hirsch, J. S., Narasimhan, M., Crawford, J. M., McGinn, T., Davidson, K. W., Barnaby, D. P., Becker, L. B., Chelico, J. D., Cohen, S. L., Cookingham, J., Coppa, K., Diefenbach, M. A., Dominello, A. J., Duer-Hefele, J., Falzon, L., Gitlin, J., Hajizadeh, N., Harvin, T. G., ... Zanos, T. P. (2020). Presenting Characteristics, Comorbidities, and Outcomes among 5700 Patients Hospitalized with COVID-19 in the New York City Area. JAMA - Journal of the American Medical Association, 323(20), 2052-2059. https://doi.org/10.1001/jama.2020.6775

5. Li, X., Xu, S., Yu, M., Wang, K., Tao, Y., Zhou, Y., Shi, J., Zhou, M., Wu, B., Yang, Z., Zhang, C., Yue, J., Zhang, Z., Renz, H., Liu, X., Xie, J., Xie, M., \& Zhao, J. (2020). Risk factors for severity and mortality in adult COVID-19 inpatients in Wuhan. Journal of Allergy and Clinical Immunology, 146(1), 110-118. https://doi.org/10.1016/j.jaci.2020.04.006

6. Halpin, D. M. G., Faner, R., Sibila, O., Badia, J. R., \& Agusti, A. (2020). Do chronic respiratory diseases or their treatment affect the risk of SARS-CoV-2 infection? In The Lancet Respiratory Medicine (Vol. 8, Issue 5, pp. 436-438). Lancet Publishing Group. https://doi.org/10.1016/S2213-2600(20)30167-3

7. Panettieri, R. A., Carson, J., Horton, D., Barrett, E., Roy, J., \& Radbel, J. (2020). Asthma and COVID: What Are the Important Questions? In Journal of Allergy and Clinical Immunology: In Practice (Vol. 8, Issue 8, pp. 2487-2488). American Academy of Allergy, Asthma and Immunology. https://doi.org/10.1016/j.jaip.2020.06.008

8. Williamson, E. J., Walker, A. J., Bhaskaran, K., Bacon, S., Bates, C., Morton, C. E., Curtis, H. J., Mehrkar, A., Evans, D., Inglesby, P., Cockburn, J., McDonald, H. I., MacKenna, B., Tomlinson, L., Douglas, I. J., Rentsch, C. T., Mathur, R., Wong, A. Y. S., Grieve, R., ... Goldacre, B. (2020). Factors associated with COVID-19-related death using OpenSAFELY. Nature, 584(7821), 430-436. https://doi.org/10.1038/s41586-0202521-4

9. Yang, J. M., Koh, H. Y., Moon, S. Y., Yoo, I. K., Ha, E. K., You, S., Kim, S. Y., Yon, D. K., \& Lee, S. W. (2020). Allergic disorders and susceptibility to and severity of COVID19: A nationwide cohort study. Journal of Allergy and Clinical Immunology, 146(4), 790-798. https://doi.org/10.1016/j.jaci.2020.08.008

10. Chhiba, K. D., Patel, G. B., Vu, T. H. T., Chen, M. M., Guo, A., Kudlaty, E., Mai, Q., Yeh, C., Muhammad, L. N., Harris, K. E., Bochner, B. S., Grammer, L. C., Greenberger, P. A., Kalhan, R., Kuang, F. L., Saltoun, C. A., Schleimer, R. P., Stevens, W. W., \& Peters, A. T. (2020). Prevalence and characterization of asthma in hospitalized and nonhospitalized patients with COVID-19. Journal of Allergy and Clinical Immunology, 146(2), 307-314.e4. https://doi.org/10.1016/j.jaci.2020.06.010 
11. Lovinsky-Desir, S., Deshpande, D. R., De, A., Murray, L., Stingone, J. A., Chan, A., Patel, N., Rai, N., DiMango, E., Milner, J., \& Kattan, M. (2020). Asthma among hospitalized patients with COVID-19 and related outcomes. Journal of Allergy and Clinical Immunology, 146(5), 1027-1034.e4. https://doi.org/10.1016/j.jaci.2020.07.026

12. Zhu, Z., Hasegawa, K., Ma, B., Fujiogi, M., Camargo, C. A., \& Liang, L. (2020). Association of asthma and its genetic predisposition with the risk of severe COVID-19. Journal of Allergy and Clinical Immunology, 146(2), 327-329.e4. https://doi.org/10.1016/j.jaci.2020.06.001

13. Docherty, A. B., Harrison, E. M., Green, C. A., Hardwick, H. E., Pius, R., Norman, L., Holden, K. A., Read, J. M., Dondelinger, F., Carson, G., Merson, L., Lee, J., Plotkin, D., Sigfrid, L., Halpin, S., Jackson, C., Gamble, C., Horby, P. W., Nguyen-Van-Tam, J. S., ... Semple, M. G. (2020). Features of 20133 UK patients in hospital with covid-19 using the ISARIC WHO Clinical Characterisation Protocol: Prospective observational cohort study. The BMJ, 369. https://doi.org/10.1136/bmj.m1985

14. Goyal, P., Choi, J. J., Pinheiro, L. C., Schenck, E. J., Chen, R., Jabri, A., Satlin, M. J., Campion, T. R., Nahid, M., Ringel, J. B., Hoffman, K. L., Alshak, M. N., Li, H. A., Wehmeyer, G. T., Rajan, M., Reshetnyak, E., Hupert, N., Horn, E. M., Martinez, F. J., ... Safford, M. M. (2020). Clinical Characteristics of Covid-19 in New York City. New England Journal of Medicine, 382(24), 2372-2374.

https://doi.org/10.1056/nejmc2010419

15. Cummings, M. J., Baldwin, M. R., Abrams, D., Jacobson, S. D., Meyer, B. J., Balough, E. M., Aaron, J. G., Claassen, J., Rabbani, L. R. E., Hastie, J., Hochman, B. R., SalazarSchicchi, J., Yip, N. H., Brodie, D., \& O’Donnell, M. R. (2020). Epidemiology, clinical course, and outcomes of critically ill adults with COVID-19 in New York City: a prospective cohort study. The Lancet, 395(10239), 1763-1770. https://doi.org/10.1016/S0140-6736(20)31189-2

16. Castro-Rodriguez, J. A., \& Forno, E. (2020). Asthma and COVID-19 in children: A systematic review and call for data. Pediatric Pulmonology, 55(9), 2412-2418. https://doi.org/10.1002/ppul.24909

17. Broadhurst, R., Peterson, R., Wisnivesky, J. P., Federman, A., Zimmer, S. M., Sharma, S., Wechsler, M., \& Holguin, F. (2020). Asthma in COVID-19 hospitalizations: An overestimated risk factor? In Annals of the American Thoracic Society (Vol. 17, Issue 12, pp. 1645-1648). American Thoracic Society. https://doi.org/10.1513/AnnalsATS.202006-613RL

18. Wang, L., Foer, D., Bates, D. W., Boyce, J. A., \& Zhou, L. (2020). Risk factors for hospitalization, intensive care, and mortality among patients with asthma and COVID19. Journal of Allergy and Clinical Immunology, 146(4), 808-812. https://doi.org/10.1016/j.jaci.2020.07.018

19. Asthma and COVID-19: scientific brief, 19 April 2021. (n.d.). Retrieved June 7, 2021, from https://www.who.int/publications/i/item/who-2019-ncov-sci-brief-asthma-2021.1

20. Welch, V., Petticrew, M., Tugwell, P., Moher, D., O’Neill, J., Waters, E., White, H., Atun, R., Awasthi, S., Barbour, V., Bhutta, Z., Cuervo, L. G., Grimshaw, J., Groves, T., Koehlmoos-Perez, T., Kristjansson, E., O’Neill, J., Oxman, A., Pantoja, T., ... Wells, G. (2012). PRISMA-Equity 2012 Extension: Reporting Guidelines for Systematic Reviews with a Focus on Health Equity. PLoS Medicine, 9(10). https://doi.org/10.1371/journal.pmed.1001333

21. Hartmann-Boyce, J., Otunla, A., Suklan, J., Schofield, E., Gunnell, J., Mighiu, A., Hobbs, R., \& Fmedsci, N. (n.d.). Risks of and from SARS-COV-2 (COVID-19) infection in people with asthma: a rapid evidence review undertaken on behalf of the World Health Organization (WHO). 
22. Ottawa Hospital Research Institute. (n.d.). Retrieved June 7, 2021, from http://www.ohri.ca/programs/clinical_epidemiology/oxford.asp

23. Guyatt, G. H., Oxman, A. D., Vist, G. E., Kunz, R., Falck-Ytter, Y., Alonso-Coello, P., \& Schünemann, H. J. (2008). GRADE: an emerging consensus on rating quality of evidence and strength of recommendations. BMJ, 336(7650), 924-926. https://doi.org/10.1136/BMJ.39489.470347.AD

24. RevMan | Cochrane Training. (n.d.). Retrieved June 15, 2021, from https://training.cochrane.org/online-learning/core-software-cochrane-reviews/revman

25. Chapter 6: Choosing effect measures and computing estimates of effect $\mid$ Cochrane Training. (n.d.). Retrieved June 7, 2021, from https://training.cochrane.org/handbook/current/chapter-06

26. 9.5.2 Identifying and measuring heterogeneity. (n.d.). Retrieved June 15, 2021, from https://handbook-5-

1.cochrane.org/chapter_9/9_5_2_identifying_and_measuring_heterogeneity.htm

27. Chapter 12: Synthesizing and presenting findings using other methods $\mid$ Cochrane Training. (n.d.). Retrieved July 1, 2021, from https://training.cochrane.org/handbook/current/chapter-12

28. Abrams, M. P., Wan, E. Y., Waase, M. P., Morrow, J. P., Dizon, J. M., Yarmohammadi, H., Berman, J. P., Rubin, G. A., Kushnir, A., Poterucha, T. J., Elias, P. A., Rubin, D. A., Ehlert, F., Biviano, A., Uriel, N., Garan, H., \& Saluja, D. (2020). Clinical and cardiac characteristics of COVID-19 mortalities in a diverse New York City Cohort. Journal of Cardiovascular Electrophysiology, 31(12), 3086-3096. https://doi.org/10.1111/jce.14772

29. Adrish, M., Chilimuri, S., Sun, H., Mantri, N., Yugay, A., \& Zahid, M. (2020). The Association of Renin-Angiotensin-Aldosterone System Inhibitors With Outcomes Among a Predominantly Ethnic Minority Patient Population Hospitalized With COVID19: The Bronx Experience. Cureus, 12(9). https://doi.org/10.7759/cureus.10217

30. Atkins, J. L., Masoli, J. A. H., Delgado, J., Pilling, L. C., Kuo, C. L., Kuchel, G. A., \& Melzer, D. (2020). Preexisting Comorbidities Predicting COVID-19 and Mortality in the UK Biobank Community Cohort. Journals of Gerontology - Series A Biological Sciences and Medical Sciences, 75(11), 2224-2230. https://doi.org/10.1093/gerona/glaa183

31. Barroso, B., Valverde-Monge, M., Cañas Jose, A., Rodrigo-Muñoz, J. M., GonzalezCano, B., Villalobos-Violan, V., Betancor, D., Gomez-Cardeñosa, A., VallejoChamorro, G., Baptista-Serna, L., Villalobos-Vilda, C., Ortega-Martin, L., GómezLópez, A., Sanchez-Pernaute, O., Romero-Bueno, F., Rodriguez-Nieto, M. J., del Pozo, V., \& Sastre, J. (2020). Prevalence, characteristics, and outcome of asthmatic patients with type 2 diseases in hospitalized patients with COVID-19 in Madrid, Spain. In Journal of Investigational Allergology and Clinical Immunology (Vol. 30, Issue 5, pp. 382-384). ESMON Publicidad S.A. https://doi.org/10.18176/jiaci.0627

32. Beurnier, A., Jutant, E. M., Jevnikar, M., Boucly, A., Pichon, J., Preda, M., Frank, M., Laurent, J., Richard, C., Monnet, X., Duranteau, J., Harrois, A., Chaumais, M. C., Bellin, M. F., Noël, N., Bulifon, S., Jaïs, X., Parent, F., Seferian, A., ... Humbert, M. (2020). Characteristics and outcomes of asthmatic patients with COVID-19 pneumonia who require hospitalisation. European Respiratory Journal, 56(5). https://doi.org/10.1183/13993003.01875-2020

33. Bozek, A., \& Winterstein, J. (2020). Montelukast's ability to fight COVID-19 infection. In Journal of Asthma. Taylor and Francis Ltd. https://doi.org/10.1080/02770903.2020.1786112 
34. Choi, Y. J., Park, J.-Y., Lee, H. S., Suh, J., Song, Y., Kwang Byun, M., Cho, J. H., Kim, J., Lee, J.-H., Park, J.-W., \& Park, H. J. (n.d.). Early View Effect of Asthma and Asthma Medication on the Prognosis of Patients with COVID-19. https://doi.org/10.1183/13993003.02226-2020

35. García-Pachón, E., Zamora-Molina, L., Soler-Sempere, M. J., Baeza-Martínez, C., GrauDelgado, J., Padilla-Navas, I., \& Gutiérrez, F. (2020). Asthma and COPD in hospitalized COVID-19 patients. Archivos de Bronconeumología (English Edition), 56(9), 604-606. https://doi.org/10.1016/j.arbr.2020.05.004

36. Grandbastien, M., Piotin, A., Godet, J., Abessolo-Amougou, I., Ederlé, C., Enache, I., Fraisse, P., Tu Hoang, T. C., Kassegne, L., Labani, A., Leyendecker, P., Manien, L., Marcot, C., Pamart, G., Renaud-Picard, B., Riou, M., Doyen, V., Kessler, R., FafiKremer, S., ... de Blay, F. (2020). SARS-CoV-2 Pneumonia in Hospitalized Asthmatic Patients Did Not Induce Severe Exacerbation. Journal of Allergy and Clinical Immunology: In Practice, 8(8), 2600-2607. https://doi.org/10.1016/j.jaip.2020.06.032

37. Keswani, A., Dhana, K., Rosenthal, J. A., Moore, D., \& Mahdavinia, M. (2020). Atopy is predictive of a decreased need for hospitalization for coronavirus disease 2019. Annals of Allergy, Asthma and Immunology, 125(4), 479-481. https://doi.org/10.1016/j.anai.2020.07.012

38. Kim, S., Jung, C. G., Lee, J. Y., Kim, G., Choi, S. W., Jin, H. J., \& Park, H. K. (2021). Characterization of asthma and risk factors for delayed SARS-CoV-2 clearance in adult COVID-19 inpatients in Daegu. In Allergy: European Journal of Allergy and Clinical Immunology (Vol. 76, Issue 3, pp. 918-921). Blackwell Publishing Ltd. https://doi.org/10.1111/all.14609

39. Ko, J. Y., Danielson, M. L., Town, M., Derado, G., Greenlund, K. J., Kirley, P. D., Alden, N. B., Yousey-Hindes, K., Anderson, E. J., Ryan, P. A., Kim, S., Lynfield, R., Torres, S. M., Barney, G. R., Bennett, N. M., Sutton, M., Talbot, H. K., Hill, M., Hall, A. J., ... George, A. (2021). Risk Factors for Coronavirus Disease 2019 (COVID-19)Associated Hospitalization: COVID-19-Associated Hospitalization Surveillance Network and Behavioral Risk Factor Surveillance System. Clinical Infectious Diseases, 72(11), e695-e703. https://doi.org/10.1093/cid/ciaa1419

40. Lemus Calderon, J. A., Beneyto Martin, P., Guzmán Rodriguez, R., Caligaris Cataldi, H. S., \& Senent Sánchez, C. J. (2021). Differentiating characteristics of patients with asthma in the severe acute respiratory syndrome coronavirus 2 infection. Annals of Allergy, Asthma and Immunology, 126(1), 92-93. https://doi.org/10.1016/j.anai.2020.09.004

41. Lieberman-Cribbin, W., Rapp, J., Alpert, N., Tuminello, S., \& Taioli, E. (2020). The Impact of Asthma on Mortality in Patients With COVID-19. Chest, 158(6), 2290-2291. https://doi.org/10.1016/j.chest.2020.05.575

42. Lombardi, C., Roca, E., Bigni, B., Cottini, M., \& Passalacqua, G. (2020). Clinical course and outcomes of patients with asthma hospitalized for severe acute respiratory syndrome coronavirus 2 pneumonia: A single-center, retrospective study. Annals of Allergy, Asthma and Immunology, 125(6), 707-709. https://doi.org/10.1016/j.anai.2020.07.029

43. Mahdavinia, M., Foster, K. J., Jauregui, E., Moore, D., Adnan, D., Andy-Nweye, A. B., Khan, S., \& Bishehsari, F. (2020). Asthma prolongs intubation in COVID-19. Journal of Allergy and Clinical Immunology: In Practice, 8(7), 2388-2391. https://doi.org/10.1016/j.jaip.2020.05.006

44. Matucci, A., Caminati, M., Vivarelli, E., Vianello, A., Micheletto, C., Menzella, F., Crisafulli, E., Passalacqua, G., Bagnasco, D., Lombardi, C., Parronchi, P., Crivellaro, M. A., Chieco-Bianchi, F., Rita Marchi, M., Guarnieri, G., Cosmi, L., Rossi, O., Almerigogna, F., Senna, G., \& Vultaggio, A. (2021). COVID-19 in severe asthmatic 
patients during ongoing treatment with biologicals targeting type 2 inflammation: Results from a multicenter Italian survey. In Allergy: European Journal of Allergy and Clinical Immunology (Vol. 76, Issue 3, pp. 871-874). Blackwell Publishing Ltd. https://doi.org/10.1111/all.14516

45. Niquini, R. P., Lana, R. M., Pacheco, A. G., Cruz, O. G., Coelho, F. C., Carvalho, L. M., Villela, D. A. M., da Costa Gomes, M. F., \& Bastos, L. S. (2020). Description and comparison of demographic characteristics and comorbidities in SARI from COVID-19, SARI from influenza, and the Brazilian general population. Cadernos de Saude Publica, 36(7). https://doi.org/10.1590/0102-311X00149420

46. NYSTAD, W., HJELLVIK, V., LARSEN, I. K., ARIANSEN, I., HELLAND, E., JOHANSEN, K. I., GULBRANDSEN, J., GULSETH, H. L., EIDE, H. N., HABERG, S. E., KARLSTAD, Y., \& BAKKEN, I. J. (2020). Underliggende tilstander hos voksne med covid-19. Tidsskrift for Den Norske Laegeforening, 140(13). https://doi.org/10.4045/tidsskr.20.0512

47. Ruano, F. J., Somoza Álvarez, M. L., Haroun-Díaz, E., Vázquez de la Torre, M., López González, P., Prieto-Moreno, A., Torres Rojas, I., Cervera García, M. D., Pérez Alzate, D., Blanca-López, N., \& Canto Díez, G. (2020). Impact of the COVID-19 pandemic in children with allergic asthma. Journal of Allergy and Clinical Immunology: In Practice, 8(9), 3172-3174.e1. https://doi.org/10.1016/j.jaip.2020.07.019

48. Santos, M. M., Lucena, E. E. S., Lima, K. C., Brito, A. A. C., Bay, M. B., \& Bonfada, D. (2020). Survival and predictors of deaths of patients hospitalized due to COVID-19 from a retrospective and multicenter cohort study in Brazil. Epidemiology and Infection, 148. https://doi.org/10.1017/S0950268820002034

49. Schultze, A., Walker, A. J., MacKenna, B., Morton, C. E., Bhaskaran, K., Brown, J. P., Rentsch, C. T., Williamson, E., Drysdale, H., Croker, R., Bacon, S., Hulme, W., Bates, C., Curtis, H. J., Mehrkar, A., Evans, D., Inglesby, P., Cockburn, J., McDonald, H. I., ... Goldacre, B. (2020). Risk of COVID-19-related death among patients with chronic obstructive pulmonary disease or asthma prescribed inhaled corticosteroids: an observational cohort study using the OpenSAFELY platform. The Lancet Respiratory Medicine, 8(11), 1106-1120. https://doi.org/10.1016/S2213-2600(20)30415-X

50. Song, J., Zeng, M., Wang, H., Qin, C., Hou, H. Y., Sun, Z. Y., Xu, S. P., Wang, G. P., Guo, C. L., Deng, Y. K., Wang, Z. C., Ma, J., Pan, L., Liao, B., Du, Z. H., Feng, Q. M., Liu, Y., Xie, J. G., \& Liu, Z. (2021). Distinct effects of asthma and COPD comorbidity on disease expression and outcome in patients with COVID-19. Allergy: European Journal of Allergy and Clinical Immunology, 76(2), 483-496. https://doi.org/10.1111/all.14517

51. Toussie, D., Voutsinas, N., Finkelstein, M., Cedillo, M. A., Manna, S., Maron, S. Z., Jacobi, A., Chung, M., Bernheim, A., Eber, C., Concepcion, J., Fayad, Z. A., \& Gupta, Y. S. (2020). Clinical and chest radiography features determine patient outcomes in young and middle-aged adults with COVID-19. Radiology, 297(1), E197-E206. https://doi.org/10.1148/radiol.2020201754

52. Thompson, C. N., Baumgartner, J., Pichardo, C., Toro, B., Li, L., Arciuolo, R., Chan, P. Y., Chen, J., Culp, G., Davidson, A., Devinney, K., Dorsinville, A., Eddy, M., English, M., Fireteanu, A. M., Graf, L., Geevarughese, A., Greene, S. K., Guerra, K., ... Fine, A. (2020). COVID-19 Outbreak - New York City, February 29-June 1, 2020. MMWR. Morbidity and Mortality Weekly Report, 69(46), 1725-1729. https://doi.org/10.15585/mmwr.mm6946a2

53. Gaudart, J., Landier, J., Huiart, L., Legendre, E., Lehot, L., Bendiane, M. K., Chiche, L., Petitjean, A., Mosnier, E., Kirakoya-Samadoulougou, F., Demongeot, J., Piarroux, R., \& Rebaudet, S. (2021). Factors associated with the spatial heterogeneity of the first wave 
of COVID-19 in France: a nationwide geo-epidemiological study. The Lancet Public Health, 6(4), e222-e231. https://doi.org/10.1016/S2468-2667(21)00006-2

54. Abate, S. M., Ali, S. A., Mantfardo, B., \& Basu, B. (2020). Rate of intensive care unit admission and outcomes among patients with coronavirus: A systematic review and Meta-analysis. PLoS ONE, 15(7 July). https://doi.org/10.1371/journal.pone.0235653

55. Zhu, Z., Hasegawa, K., Ma, B., Fujiogi, M., Camargo, C. A., \& Liang, L. (2020). Association of asthma and its genetic predisposition with the risk of severe COVID-19. Journal of Allergy and Clinical Immunology, 146(2), 327-329.e4. https://doi.org/10.1016/j.jaci.2020.06.001

56. Cao, P., Song, Y., Zhuang, Z., Ran, J., Xu, L., Geng, Y., Han, L., Zhao, S., Qin, J., He, D., Wu, F., \& Yang, L. (2021). Obesity and COVID-19 in Adult Patients With Diabetes. Diabetes, 70(5), 1061-1069. https://doi.org/10.2337/DB20-0671

57. Al-Sabah, S., Al-Haddad, M., Al-Youha, S., Jamal, M., \& Almazeedi, S. (2020). COVID19: Impact of obesity and diabetes on disease severity. Clinical Obesity, 10(6), e12414. https://doi.org/10.1111/COB.12414

58. Chowdhury, T. A. (2021). Diabetes and COVID-19: Diseases of racial, social and glucose intolerance. World Journal of Diabetes, 12(3), 198. https://doi.org/10.4239/WJD.V12.I3.198

59. Singanayagam, A., \& Johnston, S. L. (2020). Long-term impact of inhaled corticosteroid use in asthma and chronic obstructive pulmonary disease (COPD): Review of mechanisms that underlie risks. In Journal of Allergy and Clinical Immunology (Vol. 146, Issue 6, pp. 1292-1294). Mosby Inc. https://doi.org/10.1016/j.jaci.2019.12.907

60. Jeon, S., Ko, M., Lee, J., Choi, I., Byun, S. Y., Park, S., Shum, D., \& Kim, S. (2020). Identification of antiviral drug candidates against SARS-CoV-2 from FDA-approved drugs. Antimicrobial Agents and Chemotherapy, 64(7). https://doi.org/10.1128/AAC.00819-20

61. Yu, L.-M., Bafadhel, M., Dorward, J., \& Hayward, G. (2021). Inhaled budesonide for COVID-19 in people at higher risk of adverse outcomes in the community: interim analyses from the PRINCIPLE trial PRINCIPLE Collaborative Group*. MedRxiv, 2021.04.10.21254672. https://doi.org/10.1101/2021.04.10.21254672

62. COVID-19: information for the respiratory community | British Thoracic Society| Better lung health for all. (n.d.). Retrieved June 27, 2021, from https://www.britthoracic.org.uk/covid-19/covid-19-information-for-the-respiratory-community/

63. Kavanagh, J., Jackson, D. J., \& Kent, B. D. (2019). Over-and under-diagnosis in asthma. In Breathe (Vol. 15, Issue 1, pp. e20-e27). European Respiratory Society. https://doi.org/10.1183/20734735.0362-2018

64. Scott, S., Currie, J., Albert, P., Calverley, P., \& Wilding, J. P. H. (2012). Risk of misdiagnosis, health-related quality of life, and BMI in patients who are overweight with doctor-diagnosed asthma. Chest, 141(3), 616-624. https://doi.org/10.1378/chest.11-0948

65. Izcovich, A., Ragusa, M. A., Tortosa, F., Marzio, M. A. L., Agnoletti, C., Bengolea, A., Ceirano, A., Espinosa, F., Saavedra, E., Sanguine, V., Tassara, A., Cid, C., Catalano, H. N., Agarwal, A., Foroutan, F., \& Rada, G. (2020). Prognostic factors for severity and mortality in patients infected with COVID-19: A systematic review. PLoS ONE, 15(11 November). https://doi.org/10.1371/journal.pone.0241955

66. Hartling, L., Milne, A., Hamm, M. P., Vandermeer, B., Ansari, M., Tsertsvadze, A., \& Dryden, D. M. (2013). Testing the Newcastle Ottawa Scale showed low reliability between individual reviewers. Journal of Clinical Epidemiology, 66(9), 982-993. https://doi.org/10.1016/J.JCLINEPI.2013.03.003 
67. Stang, A. (2010). Critical evaluation of the Newcastle-Ottawa scale for the assessment of the quality of nonrandomized studies in meta-analyses. European Journal of Epidemiology 2010 25:9, 25(9), 603-605. https://doi.org/10.1007/S10654-010-9491-Z

68. Yelin, D., Wirtheim, E., Vetter, P., Kalil, A. C., Bruchfeld, J., Runold, M., Guaraldi, G., Mussini, C., Gudiol, C., Pujol, M., Bandera, A., Scudeller, L., Paul, M., Kaiser, L., \& Leibovici, L. (2020). Long-term consequences of COVID-19: research needs. In The Lancet Infectious Diseases (Vol. 20, Issue 10, pp. 1115-1117). Lancet Publishing Group. https://doi.org/10.1016/S1473-3099(20)30701-5

69. Chhiba, K. D., Patel, G. B., Vu, T. H. T., Chen, M. M., Guo, A., Kudlaty, E., Mai, Q., Yeh, C., Muhammad, L. N., Harris, K. E., Bochner, B. S., Grammer, L. C., Greenberger, P. A., Kalhan, R., Kuang, F. L., Saltoun, C. A., Schleimer, R. P., Stevens, W. W., \& Peters, A. T. (2020). Prevalence and characterization of asthma in hospitalized and nonhospitalized patients with COVID-19. Journal of Allergy and Clinical Immunology, 146(2), 307-314.e4. https://doi.org/10.1016/j.jaci.2020.06.010 
Table 1. Data from primary studies on whether COVID-19 outcomes in PWA differ based on subgroup characteristics. Arrows indicate direction of association; double arrows indicate statistical significance.

Abbreviations: ICU, Intensive Care Unit; LABA, long acting beta-agonsit; SABA, short acting beta-agonist; ICS, inhaled corticosteroids; OR, Odds Ratio; COPD, chronic obstructive pulmonary disease.

\begin{tabular}{|c|c|c|c|}
\hline Study ID & Age & Medication & $\begin{array}{c}\text { Asthma severity/Type/Co- } \\
\text { morbidities }\end{array}$ \\
\hline Bozek $2020^{33}$ & & $\begin{array}{c}\text { Hospitalisation } \\
\downarrow \text { Montelukast }\end{array}$ & \\
\hline Chhiba $2020^{69}$ & & $\begin{array}{c}\text { ICU admission } \\
\uparrow \text { ICS }\end{array}$ & \\
\hline Choi $2020^{34}$ & & $\begin{array}{c}\text { ICU admission } \\
\uparrow \text { ICS } \\
\downarrow \text { LABA } \\
\downarrow \text { SABA } \\
\leftrightarrow \text { LAMA } \\
\text { Mortality } \\
\uparrow \text { ICS } \\
\leftrightarrow \text { LABA } \\
\uparrow \text { SABA } \\
\downarrow \text { LAMA }\end{array}$ & $\begin{array}{c}\text { Mortality: } \\
\text { Asthma severity } \leftrightarrow\end{array}$ \\
\hline $\operatorname{Kim} 2020^{38}$ & $\begin{array}{c}\text { Mortality } \\
\text { Association between } \\
\text { asthma and mortality } \uparrow \\
\text { with increasing age }\end{array}$ & & \\
\hline Keswani $2020^{37}$ & & & $\begin{array}{c}\text { ICU admission } \\
\downarrow \text { Allergic asthma (compared to non- } \\
\text { allergic asthma) }\end{array}$ \\
\hline
\end{tabular}




\begin{tabular}{|c|c|c|c|}
\hline Mahdavinia $2020^{43}$ & $\begin{array}{c}\text { OR for association } \\
\text { between asthma and } \\
\text { hospitalisation } \uparrow \text { with } \\
\text { increasing age } \\
\text { OR for association } \\
\text { between asthma and ICU } \\
\text { admission less clear by age } \\
\text { (association } \uparrow \text { is those aged } \\
50-64 \text { compared to those } \\
\text { aged } 18-49 \text { or } 65+\text { ) }\end{array}$ & & \\
\hline Schultze $2020^{49}$ & & $\begin{array}{c}\text { Mortality } \\
\text { Compared to SABA: } \\
\uparrow \text { low/medium dose ICS } \\
\uparrow \uparrow \text { high dose ICS }\end{array}$ & \\
\hline Wang $2020^{18}$ & 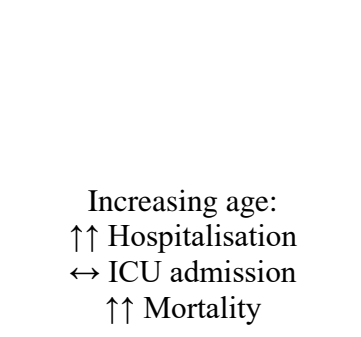 & $\begin{array}{c}\text { Hospitalisation: } \\
\downarrow \text { ICS } \\
\uparrow \text { ICS-LABA } \\
\text { ICU admission: } \\
\downarrow \text { ICS } \\
\downarrow \text { ICS-LABA } \\
\text { Mortality: } \\
\downarrow \text { ICS } \\
\downarrow \text { ICS-LABA }\end{array}$ & $\begin{array}{c}\text { Hospitalisation: } \\
\uparrow \uparrow \text { COPD } \\
\text { ICU admission: } \\
\uparrow \text { COPD } \\
\text { Mortality: } \\
\uparrow \text { COPD }\end{array}$ \\
\hline Williamson $2020^{8}$ & & $\begin{array}{l}\text { Mortality } \\
\uparrow \text { OCS use }\end{array}$ & \\
\hline
\end{tabular}




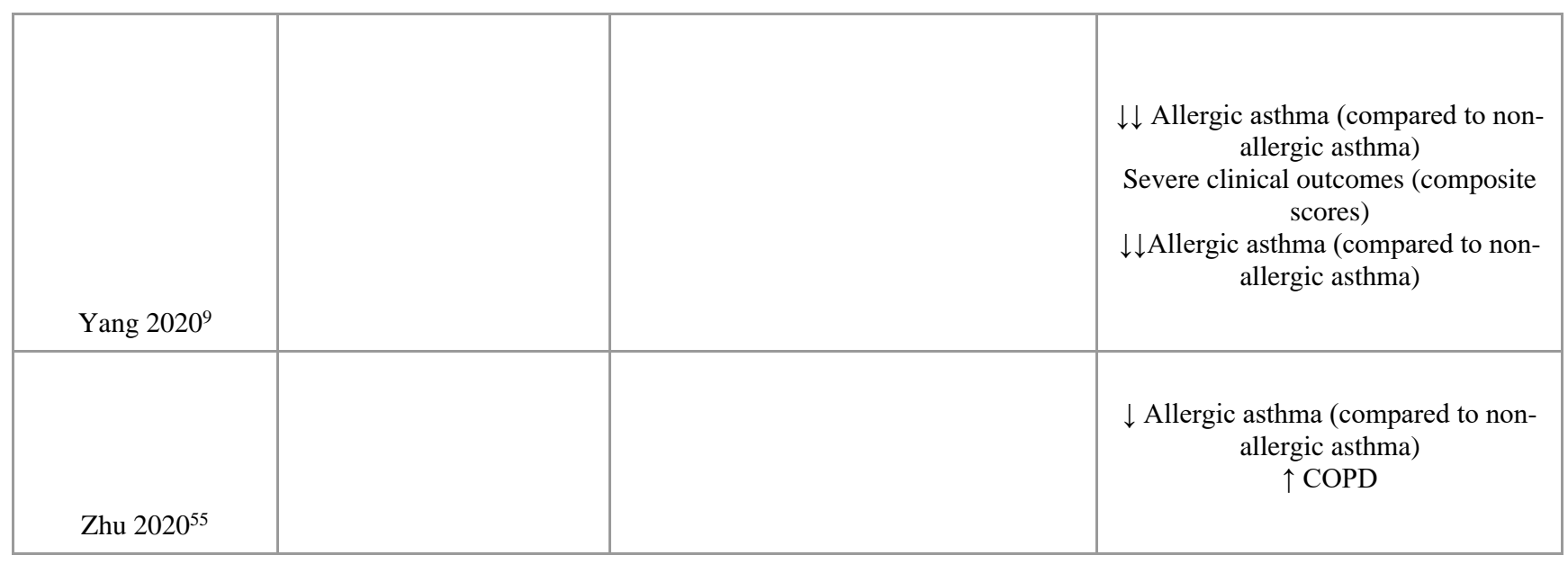


Table 2. Summary of findings table: Risks of infection, hospital and ICU admission, and death from COVID-19 in people with asthma compared to people without asthma

\begin{tabular}{|c|c|c|c|}
\hline Outcome & $\begin{array}{l}\text { Number of } \\
\text { participants } \\
\text { (studies) }\end{array}$ & $\begin{array}{l}\text { Relative } \\
\text { effect }(95 \% \\
\text { CI) }\end{array}$ & Certainty \\
\hline $\begin{array}{l}\text { Prevalence: as a proxy for risk of } \\
\text { infection }\end{array}$ & $\begin{array}{l}83,294 \\
\text { (21 observational } \\
\text { studies) }\end{array}$ & $\begin{array}{l}\text { PP: } 9.38 \% \\
\text { (7.38 to } 11.38)\end{array}$ & $\begin{array}{l}\oplus \bigcirc \bigcirc \bigcirc \\
\text { VERY LOW }\end{array}$ \\
\hline Mortality & $\begin{array}{l}62,521 \\
\text { ( } 15 \text { observational } \\
\text { studies) }\end{array}$ & $\begin{array}{l}\text { OR: 0.9 } \\
(0.72-1.13)\end{array}$ & $\begin{array}{l}\oplus \bigcirc \bigcirc \bigcirc \\
\text { VERY LOW }\end{array}$ \\
\hline Hospitalization & $\begin{array}{l}25,065 \\
\text { ( } 9 \text { observational } \\
\text { studies) }\end{array}$ & $\begin{array}{l}\text { OR: 0.95 } \\
(0.71-1.26)\end{array}$ & $\begin{array}{l}\oplus \bigcirc \bigcirc \bigcirc \\
\text { VERY LOW }\end{array}$ \\
\hline ICU admission & $\begin{array}{l}13,092 \\
\text { ( } 9 \text { observational } \\
\text { studies) }\end{array}$ & $\begin{array}{l}\text { OR: } \mathbf{0 . 9 6} \\
(0.75-1.24)\end{array}$ & $\begin{array}{l}\oplus \bigcirc \bigcirc \bigcirc \\
\text { VERY LOW }\end{array}$ \\
\hline
\end{tabular}

PP: Pooled Prevalence; CI: Confidence interval; OR: Odds ratio ; SD: Standard deviation

1 - Downgraded two levels due to unexplained statistical heterogeneity, one level due to possible publication bias (skewed funnel plot).

2 - Downgraded one level due to unexplained statistical heterogeneity, downgraded one level due to imprecision (wide CIs).

3 - Downgraded two levels due to unexplained statistical heterogeneity, downgraded one level due to imprecision (wide CIs)

4 - Downgraded one level due to imprecision (wide CIs). 